\title{
SUBCRITICALITY, POSITIVITY, AND GAUGEABILITY OF THE SCHRÖDINGER OPERATOR
}

\author{
Z. ZHAO
}

\section{INTRODUCTION}

We investigate properties of the Schrödinger operator $H:=$ $-(\Delta / 2)+V \geq 0$ in $R^{d}(d \geq 3)$ in the following three aspects:

(I) Subcriticality: Intuitively, the idea is that if $H \geq 0$ is subcritical, then it should be possible to perturb $H$ by small perturbations and still keep its nonnegativity. More precisely, we have the following assertions:

(a) For any $q \in B_{c}$ ( $B_{c}$ denotes the class of bounded Borel functions with compact support), there exists an $\varepsilon>0$ such that $-(\Delta / 2)+V+\varepsilon q \geq 0$.

(b) There exists a function $q \in B_{c}, q \leq 0$ and $q \not \equiv 0$ a.e. such that $-(\Delta / 2)+V+q \geq 0$.

There have been two other definitions of subcriticality:

(c) (B. Simon [7]) There exists $\beta>0$ such that $-(\Delta / 2)+$ $(1+\beta) V \geq 0$.

(d) (M. Murata [6]) There exists a positive Green function $G^{H}(\cdot, \cdot)$ for $H$.

(II) Strong Positivity:

(e) There exists a positive solution $u>0$ of $H u=0$ with the limit: $\lim _{|x| \rightarrow \infty} u(x)>0$.

(f) There exists a solution $u$ of $H u=0$ with $c^{\prime} \geq u \geq c>0$.

(g) There exists a solution $u$ of $H u=0$ with $u \geq c>0$.

(III) Gaugeability: Let $\left\{X_{t}: t \geq 0\right\}$ be the Brownian motion in $R^{d}$ and let $E^{x}$ denote the expectation over the Brownian paths starting from $x \in R^{d}$. Put $u_{0}(x):=E^{x}\left[\exp \left(-\int_{0}^{\infty} V(X s) d s\right)\right]$.

(h) $u_{0}(x) \not \equiv \infty$ in $R^{d}$.

(i) $u_{0}(x)$ is bounded in $R^{d}$.

Received by the editors October 25, 1989.

1980 Mathematics Subject Classification (1985 Revision). Primary 81C20. 
For any $y$ in $R^{d}$, we define the $y$-conditional Brownian motion of Doob type (see [10]) and use $E_{y}^{x}$ to denote the expectation over the $y$-conditional Brownian paths starting from $x$. Put

$$
u_{0}(x, y):=E_{y}^{x}\left[\exp \left(-\int_{0}^{\xi} V(X s) d s\right)\right], \quad x, y \in R^{d},
$$

where $\xi$ is the lifetime of the process.

(j) $u_{0}(x, y)<\infty$ for some $(x, y)$ in $R^{d} \times R^{d}, x \neq y$.

(k) $u_{0}(x, y)$ is bounded in $R^{d} \times R^{d}$.

Our main result is the equivalence of all the assertions (a) through (k) listed above for a large class of potentials $V$ given below.

\section{Restricted Kato Class $K_{d}^{\infty}$}

For a function $V$ in $K_{d}^{\mathrm{loc}}, d \geq 3$ (see [8] for definition of the Kato classes $K_{d}^{\text {loc }}$ and $K_{d}$ ), we add a similar Kato condition around the point at $\infty$ and then form a new class $K_{d}^{\infty}$ called the restricted Kato class:

(1) $K_{d}^{\infty}:=\left\{V \in K_{d}^{\mathrm{loc}}: \lim _{A \rightarrow \infty}\left[\sup _{|x| \geq A} \int_{|y| \geq A} \frac{|V(y)|}{|y-x|^{d-2}} d y\right]=0\right\}$.

It is easy to see that $K_{d} \cap L^{1}\left(R^{d}\right) \subseteq K_{d}^{\infty} \subseteq K_{d}$. It can be verified by Hölder's inequality that $K_{d}^{\infty}$ also contains the class of "short range potentials":

$$
\left\{V \in K_{d}: V(x)=O\left(|x|^{-\rho}\right) \text { as }|x| \rightarrow \infty, \rho>2\right\} .
$$

We note that Murata [5] proved some part of the above-mentioned equivalences for subcriticality for potentials satisfying the condition in (2) with $\rho>4$.

For $V \in K_{d}^{\infty}$, put $\|V\| \|:=\sup _{x \in R^{d}} \int_{R^{d}}\left(|V(y)| /|x-y|^{d-2}\right) d y<$ $\infty$. We add two more assertions to the list in (I):

(1) There exists an $\varepsilon>0$ such that for any $q \in K_{d}^{\infty}$ with $\|\mid\| q \|<\varepsilon,-(\Delta / 2)+V+q \geq 0$.

(m) There exists a function $q \in K_{d}^{\infty}, q \leq 0$ and $q \not \equiv 0$ a.e. such that $-(\Delta / 2)+V+q \geq 0$. 


\section{MAIN THEOREM AND SKETCH OF THE PROOF}

Theorem. For any $V \in K_{d}^{\infty}(d \geq 3)$, the conditions (a) through (m) are equivalent.

Sketch of the proof. Since $V \in K_{d}^{\infty}$, there exists a $r>0$ such that

$$
\sup _{|x| \geq r}\left[C_{d} \int_{|y| \geq r} \frac{|V(y)|}{|x-y|^{d-2}} d y\right]<\frac{1}{2},
$$

where $C_{d}=\Gamma((d / 2)-1) / 2 \pi^{d / 2}$. Let $D=\left\{x \in R^{d}:|x|>r\right\}$ and $B=\left\{x \in R^{d}:|x|<2 r\right\}$. Put $T:=\tau_{B}+\tau_{D} \circ \theta_{\tau_{B}}$ (the shuttle time), where $\tau_{U}$ is the exit time from a domain $U$ and $\theta$ is the shift operator on paths. We define the shuttle operator $S_{V}$ in the Banach space $C(\partial D)$ : for $f \in C(\partial D)$,

(4) $S_{V} f(x):=E^{x}\left[T<\infty ; \exp \left(-\int_{0}^{T} V(X s) d s\right) f(X(T))\right]$, $x \in \partial D$.

By Khasmin'skii's lemma together with (3) and the arguments similar to those in [10], we can prove $S_{V}$ is an integral operator with continuous kernel:

$$
S_{V} f(x)=\int_{\partial D} \Phi(x, y) f(y) \sigma(d y) \quad(\sigma \text { is the area measure }),
$$

where

$$
\begin{aligned}
& \Phi(x, y)=9(d-2)^{2} C_{d}^{2} r^{2} \\
& \times \int_{\partial D} \frac{E_{z}^{x}\left[\exp \left(-\int_{0}^{\tau_{B}} V(X s) d s\right)\right] E_{y}^{z}\left[\exp \left(-\int_{0}^{\tau_{D}} V(X s) d s\right)\right]}{|x-z|^{d}|y-z|^{d}} \sigma(d z), \\
& (x, y) \in \partial D \times \partial D .
\end{aligned}
$$

Put $\lambda(V):=\lim _{n \rightarrow \infty} \sqrt[n]{\left\|\left(S_{V}\right)^{n}\right\|}$.

Introducing the shuttle operator $S_{V}$ and its spectral radius $\lambda(V)$ is the key idea in connecting the seemingly different assertions in the list (a) through $(\mathrm{m})$. In fact, we add a new equivalent assertion as a linkage among the assertions $(\mathrm{a})$ through $(\mathrm{m})$ :

(n) $\lambda(V)<1$. 
$\lambda(V)$, as a function of $V$, has the following properties:

Lemma. (L1) If $\left\|\mid V_{n}-V\right\| \| \rightarrow$, then $\lambda\left(V_{n}\right) \rightarrow \lambda(V)$.

(L2) If $V_{1} \leq V_{2}$ and $V_{1} \not \equiv V_{2}$ a.e., then $\lambda\left(V_{1}\right)>\lambda\left(V_{2}\right)$.

Both properties are based on the integral kernel representation (5) in terms of path integrals. We also need a characterization of nonnegativity of $H$, which can be regarded as a higher dimensional version of a result by Chung and Varadhan [2].

Proposition A. For $V \in K_{d}^{\infty},-(\Delta / 2)+V \geq 0$ if and only if $\lambda(V) \leq 1$.

We now sketch the proof of some nontrivial implications in connection with $(\mathrm{n})$. (n) $\Leftrightarrow(\mathrm{h})$ : This equivalence is mainly given by the equality:

$$
E^{x}\left[\exp \left(-\int_{0}^{\infty} V(X s) d s\right)\right]=\sum_{n=0}^{\infty}\left(S_{V}\right)^{n} g(x), \quad x \in \partial D
$$

where $g(x):=E^{x}\left[T=\infty ; \exp \left(-\int_{0}^{T} V(X s) d s\right)\right]$. The idea behind the equality (6) is that almost every Brownian path in $R^{d}(d \geq 3)$ will shuttle finitely many times between $\partial B$ and $\partial D$ before it goes off to $\infty$.

(n) $\Rightarrow(1)$ : Suppose $\lambda(V)<1$. By (L1), if $\|q\|$ is small enough, then $\lambda(V+q)<1$. Therefore $-(\Delta / 2)+V+q \geq 0$ by Proposition A.

$(\mathrm{m}) \Rightarrow(\mathrm{n})$ : By (L2) and Proposition A, we have $\lambda(V)<$ $\lambda(V+q) \leq 1$.

(c) $\Rightarrow(\mathrm{n})$ : For each $0 \leq t \leq 1+\beta$, put $f(t):=\ln [\lambda(t V)]=$ $\lim _{n \rightarrow \infty}(1 / n) \ln \left\|\left(S_{t V}\right)^{n}\right\|$.

Since for each $n, \ln \left\|\left(S_{t V}\right)^{n}\right\|$ is a convex function of $t$ by using the stopped path integral and the Cauchy-Schwarz inequality, so is the limit $f(t)$. Since $f(t) \leq 0$ in $[0,1+\beta]$ by Proposition A and $f(0)<0$ by the transient property of the Brownian motion in $R^{d}(d \geq 3)$, we obtain $f(1)<0$, i.e. $\lambda(V)<1$.

Another key idea is the connection between the Green function $G^{H}(x, y)$ and the conditional Feynman-Kac gauge (see Zhao [10]):

$$
G^{H}(x, y)=G^{\Delta / 2}(x, y) E_{y}^{x}\left[\exp \left(-\int_{0}^{\xi} V(X s) d s\right)\right] .
$$

The proof of equivalences in the list (III) involves gauge and conditional gauge arguments similar to those in [1], [3] and [9]. 


\section{ACKNOWLEDGMENTS}

The original idea about these equivalences for subcritical Schrödinger operators comes from joint work with F. Gesztesy [4] in the corresponding one-dimensional case. His inspiration and the numerous discussions with him are gratefully acknowledged.

\section{REFERENCES}

1. K. L. Chung and M. Rao, Feynman-Kac functional and the Schrödinger equation, Seminar on Stochastic Processes, Birkhäuser, Boston, 1981.

2. K. L. Chung and S. R. S. Varadhan, Kac functional and Schrödinger equation, Studia Math., T. LXVIII (1980), 249-260.

3. M. Cranston, E. Fabes, and Z. Zhao, Conditional gauge and potential theory for the Schrödinger operator, Trans. Amer. Math. Soc. 307 (1988), 171-194.

4. F. Gesztesy and Z. Zhao, On critical and subcritical Sturm-Liouville operators, J. Funct. Anal (to appear).

5. M. Murata, Positive solutions and large time behavior of Schrödinger semigroups, Simon's problem, J. Funct. Anal. 56 (1984), 300-310.

6. Structure of positive solutions to $(-\Delta+V) u=0$ in $R^{n}$, Duke Math J. 53 (1986), 869-943.

7. B. Simon, Large time behavior of the $L^{p}$ norm of Schrödinger semigroups, J. Funct. Anal. 40 (1981), 66-83.

8. __ Schrödinger semigroups, Bull. Amer. Math. Soc. 7 (1982), 447-526.

9. Z. Zhao, Conditional gauge and unbounded potential, Z. Wahrsch. Verw. Gebiete 65 (1983), 13-18.

10. __ Green function for Schrödinger operator and conditioned FeynmanKac gauge, J. Math. Anal. Appl. 116 (1986), 309-334.

Department of Mathematics, University of Missouri, Columbia, MissOURI 65211 
